Math. Model. Nat. Phenom.

Vol. 9, No. 4, 2014, pp. 227-237

DOI: $10.1051 / \mathrm{mmnp} / 20149414$

\title{
Modeling of Environmental Adaptation versus Pollution Mitigation
}

\author{
Y. Yatsenko ${ }^{1 *}$, N. Hritonenko ${ }^{2}$, T. Bréchet ${ }^{3}$ \\ ${ }^{1}$ School of Business, Houston Baptist University, 7502 Fondren, Houston, Texas 77074, USA \\ 2 Department of Mathematics, Prairie View A\&M University, Prairie View, Texas 77446, USA \\ ${ }^{3}$ Université catholique de Louvain, CORE and Louvain School of Management, Belgium
}

\begin{abstract}
The paper combines analytic and numeric tools to investigate a nonlinear optimal control problem relevant to the economics of climate change. The problem describes optimal investments into pollution mitigation and environmental adaptation at a macroeconomic level. The steady-state analysis of this problem focuses on the optimal ratio between adaptation and mitigation. In particular, we analytically prove that the long-term investments into adaptation are profitable only for economies above certain efficiency threshold. Numerical simulation is provided to estimate how the economic efficiency and capital deterioration affect the optimal policy.
\end{abstract}

Keywords and phrases: climate change, environmental adaptation, mitigation, optimal control, steady state analysis

Mathematics Subject Classification: 91B76, 34H05, 49J22, 65R2

\section{Introduction}

Optimization models play an increasing role in modern climate change studies $[1,3,11,19,22]$. One of relevant issues is to compare two major strategies to deal with climate change, namely the mitigation of greenhouse gas emissions and the adaptation to global warming. Adapting to climate change means an adjustment in the economic-environmental system that reduces the damages caused by climate change [21]. The mitigation is an anthropogenic intervention to reduce the sources (or enhance the sinks) of greenhouse gases [16]. Despite a growing importance of the mitigation and adaptation issues in environmental modeling, just a few papers analytically investigate the impact of mitigation and adaptation on long-run economic development $[1,3-6,9,15,17,20]$. We contribute to this emerging literature.

This paper extends the analysis of the economic optimization model of [4] that involved environmental pollution, mitigation, and adaptation. One of its results was the existence of a threshold of economic efficiency and environmental vulnerability under which adaptation measures were not optimal. Some numerical experiments suggested that this critical threshold might be rather low. In order to override essential technical challenges and obtain meaningful economic results, the analysis was restricted to the case with no capital deterioration (common in the economic literature). However, there are economic

${ }^{*}$ Corresponding author. E-mail: yyatsenko@hbu.edu 
reasons to be cautious about the assumption of zero capital depreciation [2,10,14], especially for longterm policies that use steady state analysis. The goal of the present paper is to consider the model of [4] in the general case, and to scrutinize effects of capital deterioration on the optimal dynamics of the modeled economy.

The paper is organized as follows. Section 2 describes and interprets the nonlinear optimal control problem under study and presents the condition for an extremum. Section 3 provides the steady-state analysis of the problem and reveals important economic implications of the obtained qualitative dynamics. Section 4 supplements analytic results with numeric simulation and demonstrates that the impact of capital deterioration is quite essential and cannot be ignored in designing optimal policies to combat climate change. Section 5 concludes.

\section{Statement of Optimal Control Problem}

We investigate the following nonlinear optimal control problem: find the unknown functions $C(t), D(t)$, $P(t), Y(t), K(t), I_{K}(t), I_{D}(t), B(t), t \in[0, \infty)$, that maximize the following functional:

$$
\max \int_{0}^{\infty} e^{-\rho t}\left(\ln C-\eta(D) \frac{P^{1+\mu}}{1+\mu}\right) d t
$$

subject to the following inequality-constraints:

$$
I_{K}(t) \geqslant 0, I_{D}(t) \geqslant 0, C(t) \geqslant 0, t \in[0, \infty),
$$

and equality-constraints:

$$
\begin{gathered}
Y(t)=A K^{\alpha}(t)=I_{K}(t)+I_{D}(t)+B(t)+C(t), \\
K^{\prime}(t)=I_{K}(t)-\delta K(t), K(0)=K_{0}, \\
D^{\prime}(t)=I_{D}(t)-\delta D(t), D(0)=D_{0}, \\
P^{\prime}(t)=-\delta_{P} P(t)+\gamma Y(t) / B(t), P(0)=P_{0} .
\end{gathered}
$$

The dynamic macroeconomic model (2.1)-(2.6) describes a one-sector economy that produces a single final product $Y$ and distributes it among the consumption $C$, the investment $I_{K}$ in physical capital $K$, the investment $I_{D}$ in environmental adaptation $D$, and the abatement investment $B$ in order to maximize the infinite horizon utility (2.1). The given $\rho>0$ is the rate of time preference and $0<\alpha<1$ is the parameter of Cobb-Douglas production function. The economic efficiency factor $A>0$ and the capital deterioration rate $\delta>0$ will play a central role in the analysis below.

The macro model (2.4)-(2.5) assumes the depreciation rate $\delta$ for physical capital $K$ and adaptation capital $D$. Indeed, both $K$ and $D$ cover short-term life capital stocks (like air-cooling systems, about 10-15 years) as well as long term infrastructures (like dams). Nevertheless, it is true that, if adaptation capital is (by definition) more resilient to climate change than standard capital, its lifetime should be longer, which can translate into a lower depreciation rate. But this assumes that we can differentiate the nature of the two capital stocks and empirically assess the difference between the two depreciation rates. It is clear that investing in a more resilient capital stock should be socially beneficial (longer capital lifetime), but assessing such an issue raises several empirical questions that are beyond the scope of this paper.

The environmental quality is characterized by the pollution stock $P$. The emission factor $\gamma>0$ in the pollution equation (2.6) reflects the environmental dirtiness of the economy and describes the pollution flow caused by economic production processes. The abatement investment $B$ in (2.6) reduces this pollution flow and, so, represents mitigation in the model. The pollution stock $P$ increases with this flow and deteriorates in time at a constant natural decay rate $\delta_{P}>0$. Having abatement $B$ in the denominator of the pollution flow function is a convenient and common modeling option, e.g., [23, 24]. 
It gives the net flow of pollution, that is, the flow resulting from productive activity net of abatement efforts, which are considered as flows. The choice of $Y / B$ is also reasonable from a practical viewpoint if we consider that a certain environmental cleanup work always takes place (like installing/replacing air filters, cleaning water drains, planting trees). More complex abatement specifications would make the steady state analysis of our model analytically intractable. Under our model specifications, the optimal steady-state $B$ is always strictly positive because the optimization problem itself rejects the choice of $B=0$. So, the pollution $P$ cannot tend to infinity in our setting, simply because abatement $B$ would never approach zero.

The utility function in the integrand of (2.1) depends on the consumption $C$, pollution $P$, and adaptation capital $D$. The parameter $\mu>0$ reflects the negative increasing marginal utility of pollution, while a decreasing function $\eta(D)$ represents the environmental vulnerability of the economy, which can be reduced by investments $D$ in adaptation.

\subsection{Extremum Conditions}

The optimal control problem (2.1)-(2.6) includes seven unknowns. Let us choose $I_{K}, I_{D}, C$ as the independent decision variables, that belong to the space $L_{\infty l o c}[0, \infty)$ of measurable functions bounded on any finite interval of $[0, \infty)[8]$. Then $K, D, B, P$ are four state variables determined from four constraints-equalities (2.3)-(2.6). If we choose $B$ as a control and $C$ as a state variable, all qualitative results of this paper remain the same. The optimality condition for this problem is obtained using the standard Hamiltonian-based technique $[7,12,18]$.

Lemma 2.1. [4] The interior optimal trajectories $K, B, C, D$, and $P$ in the problem (2.1)-(2.6) satisfy the following five nonlinear differential equations:

$$
\begin{gathered}
A K^{\alpha}=\delta K+K^{\prime}+\delta D+D^{\prime}+B+C, \\
\alpha A K^{\alpha-1}-\alpha \frac{B}{K}-\frac{C^{\prime}}{C}=\delta+\rho, \\
P^{\prime}+\delta_{P} P=\gamma \frac{A K^{\alpha}}{B}, \\
\eta(D) P^{\mu}=\frac{B}{\gamma A K^{\alpha} C}\left(B\left(\delta_{P}+\rho\right)+\alpha \frac{B}{K} K^{\prime}+\frac{B}{C} C^{\prime}-2 B\right), \\
-\eta^{\prime}(D) \frac{P^{\mu+1}}{\mu+1}-\frac{1}{C}\left(\rho+\delta+\frac{C^{\prime}}{C}\right)=0 .
\end{gathered}
$$

This lemma can be easily extended into a complete maximum principle that handles both interior and corner solutions. For example, the left-hand side of (2.11) represents the functional derivative of the functional (2.1) with respect to the unknown control $D$. However, essential technical challenges appear in solving the interior optimality conditions (2.7)-(2.11) and using them for a qualitative analysis of optimal dynamics. To override some of these challenges, [4] considers the problem (2.1)-(2.6) in the absence of capital deterioration: $\delta=0$. It allows proving the existence of a unique steady-state trajectory and finding approximate analytic expressions for the steady state, which lead to essential economic implications. At the same time, the assumption about zero capital depreciation is too restrictive, especially, during a steady-state analysis. The corresponding economic disturbances can be quite essential [2].

The goal of the present paper is to extend the outcomes of [4] to the general case of the problem (2.1)-(2.6) with capital deterioration $\delta>0$ and estimate how the capital deterioration affects the optimal dynamics. 


\section{Steady-State Analysis}

We restrict ourselves to the steady-state analysis of the optimal control problem (2.1)-(2.6). A steady state solution (if it exists) describes the long-term sustainable dynamics of the economic-environmental system under study, which is useful for policy recommendations. The steady-state analysis is the only analytic option in dynamic economic models with several endogenous controls $[12,13,22]$. Let us analyze a possibility of stationary states of the form

$$
K(t)=\bar{K}, C(t)=\bar{C}, B(t)=\bar{B}, P(t)=\bar{P}, D(t)=\bar{D}, t \in[0, \infty),
$$

in the optimization model (2.1)-(2.6). As in [4], the environmental vulnerability is expressed by the following exponential function:

$$
\eta(D)=\underline{\eta}+(\bar{\eta}-\eta) e^{-a D}, \text { with } \bar{\eta}>\underline{\eta}>0, a>0,
$$

that monotonically decreases from $\eta(0)=\bar{\eta}$ at $D=0$ to $\eta(\infty)=\eta$ when $D \rightarrow \infty$. The substitution of (3.1) and (3.2) into the differential equations (2.7)-(2.11) provides the following system of five nonlinear equations:

$$
\begin{gathered}
A \bar{K}^{\alpha}=\bar{B}+\bar{C}+\delta \bar{K}+\delta \bar{D}, \\
\alpha A \bar{K}^{\alpha-1}-\alpha \frac{\bar{B}}{\bar{K}}=\delta+\rho, \\
\delta_{P} \bar{P}=\gamma \frac{A \bar{K}^{\alpha}}{\bar{B}}, \\
\left(\underline{\eta}+(\bar{\eta}-\underline{\eta}) e^{-a \bar{D}}\right) \bar{P}^{\mu}=\frac{\bar{B}^{2}}{\gamma A \bar{K}^{\alpha} \bar{C}}\left(\delta_{P}+\rho\right), \\
a(\bar{\eta}-\underline{\eta}) e^{-a \bar{D}} \frac{\bar{P}^{\mu+1}}{\mu+1}=\frac{\delta+\rho}{\bar{C}},
\end{gathered}
$$

with respect to five unknown scalars $\bar{K}, \bar{C}, \bar{B}, \bar{P}$ and $\bar{D}$. The nonlinear system (3.3)-(3.7) reduces to two nonlinear equations with respect to $\bar{K}$ and $\bar{D}$ :

$$
\begin{gathered}
\frac{\bar{K}^{\alpha}\left(A-\bar{K}^{1-\alpha}(\delta+\rho) / \alpha\right)^{2+\mu}}{\bar{K}(\delta+\rho) / \alpha-\delta(\bar{K}+\bar{D})}=\frac{\gamma^{1+\mu} A^{1+\mu}}{\left(\delta_{P}+\rho\right) \delta_{P}^{\mu}}\left(\underline{\eta}+(\bar{\eta}-\underline{\eta}) e^{-a \bar{D}}\right), \\
a(\bar{\eta}-\underline{\eta}) e^{-a \bar{D}} \frac{\gamma^{\mu+1} A^{\mu+1}}{\delta_{P}^{\mu+1}(\mu+1)}-\frac{\rho\left(A-\bar{K}^{1-\alpha}(\delta+\rho) / \alpha\right)^{\mu+1}}{\bar{K}(\delta+\rho) / \alpha-\delta(\bar{K}+\bar{D})}=0 .
\end{gathered}
$$

The nonlinear system (3.8)-(3.9) determines the interior optimal dynamics of the problem (2.1)-(2.6). If these two equations have a solution $\bar{K} \geqslant 0$ and $\bar{D} \geqslant 0$, then the other steady state components are expressed in the terms of $\bar{K}$ and $\bar{D}$ by (3.3)-(3.7) as

$$
\begin{gathered}
\bar{B}=A \bar{K}^{\alpha}-\bar{K}(\delta+\rho) / \alpha, \\
\bar{C}=\bar{K}(\delta+\rho) / \alpha-\delta(\bar{K}+\bar{D}), \\
\bar{P}=\frac{\gamma A}{\delta_{P}\left(A-\bar{K}^{1-\alpha}(\delta+\rho) / \alpha\right)}, \\
\bar{I}_{K}=\delta \bar{K}, \bar{I}_{D}=\delta \bar{D} .
\end{gathered}
$$

We cannot analytically prove the existence of a solution $(\bar{K}, \bar{D})$ to the nonlinear equations (3.8)-(3.9). To keep the analysis tractable, some simplifying assumptions must be made. In particular, the existence of a unique solution to (3.8)-(3.9) without capital depreciation, i.e., at $\delta=0$, is described by the following lemma. 
Lemma 3.1. [4] The optimal control problem (2.1)-(2.6) at $\delta=0$ possesses a unique steady state $\bar{K}, \bar{C}$, $\bar{B}, \bar{P}, \bar{D})$ with $0<\bar{K}<(\alpha A / \rho)^{\frac{1}{1-\alpha}}$ and $\bar{D} \geqslant 0$. If $\bar{K}$ is smaller than a certain threshold value $\overline{K_{C}}>0$, then the optimal $\bar{D}=0$. Otherwise, the unique $\bar{K}>0$ and $\bar{D}>0$ are determined from the system of two equations (3.8) and (3.9).

In the case of small positive values of $\delta$, the solution to (2.1)-(2.6) should be close to the one of Lemma 2. A key outcome of Lemma 2 is that adaptation is not optimal when the capital level $\bar{K}$ is low. Here, we extend this result and prove that it holds in the general case of the problem, i.e. with capital depreciation. For the purpose of analysis, we rewrite equations (3.8)-(3.9) as

$$
f_{1}(\bar{K}, \bar{D})=0, f_{2}(\bar{K}, \bar{D})=0,
$$

where

$$
\begin{gathered}
f_{1}(\bar{K}, \bar{D})=\bar{K}^{\alpha-1}\left(A-\bar{K}^{1-\alpha} \frac{\delta+\rho}{\alpha}\right)^{2+\mu}-\frac{(\gamma A)^{1+\mu}\left(\underline{\eta}+(\bar{\eta}-\underline{\eta}) e^{-a \bar{D}}\right)}{\left(\delta_{P}+\rho\right) \delta_{P}^{\mu}}\left(\frac{\delta+\rho}{\alpha}-\delta-\delta \frac{\bar{D}}{\bar{K}}\right), \\
f_{2}(\bar{K}, \bar{D})=\frac{(\gamma A)^{\mu+1} a(\bar{\eta}-\underline{\eta}) e^{-a \bar{D}}}{\delta_{P}^{\mu+1}(\mu+1)}\left(\frac{\delta+\rho}{\alpha}-\delta-\delta \frac{\bar{D}}{\bar{K}}\right)-\rho \bar{K}^{-1}\left(A-\bar{K}^{1-\alpha} \frac{\delta+\rho}{\alpha}\right)^{1+\mu}
\end{gathered}
$$

and introduce the auxiliary nonlinear equation

$$
\frac{\left(A-\widehat{K}^{1-\alpha}(\delta+\rho) / \alpha\right)^{2+\mu}}{\widehat{K}^{1-\alpha}}=\frac{\gamma^{1+\mu} A^{1+\mu} \bar{\eta}((\delta+\rho) / \alpha-\delta)}{\left(\delta_{P}+\rho\right) \delta_{P}^{\mu}},
$$

in the unknown $\widehat{K}$, which is obtained from the equation $f_{1}(\bar{K}, \bar{D})$ at $\bar{D}=0$. The nonlinear equation (3.17) always possesses a unique solution $\widehat{K}, 0<\widehat{K}<[\alpha A /(\delta+\rho)]^{1 /(1-\alpha)}$ (see [4]). This yields the following theorem.

Theorem 3.2. If the solution $\widehat{K}$ of the equation (3.17) satisfies the inequality

$$
A \widehat{K}^{\alpha}-\widehat{K} \frac{\delta+\rho}{\alpha} \leqslant \frac{(1+\mu) \bar{\eta} \rho \delta_{P}}{a(\bar{\eta}-\underline{\eta})\left(\delta_{P}+\rho\right)}
$$

then the optimal steady-state adaptation $\bar{D}=0$ and the optimal capital level $\bar{K}=\widehat{K}$ is given by (3.17). If (3.18) does not hold, then the optimal $\bar{D}>0$ and $\bar{K}>\widehat{K}$ are found from by the system of two nonlinear equations (3.8) and (3.9).

Proof. Both equations (3.8) and (3.9) cannot be satisfied at small $\bar{K}$ and any nonnegative $\bar{D}$. Indeed, by $(3.15)-(3.16), \lim _{\bar{K} \rightarrow \infty} f_{1}(\bar{K}, \bar{D})=\infty$ and $\lim _{\bar{K} \rightarrow \infty} f_{2}(\bar{K}, \bar{D})=-\infty$ at any $\bar{D}>0$. It means that, if the optimal control $\bar{K}>0$ is small enough, then the only choice for the solution $\bar{D}$ is to be corner. As discussed in Section 2.1, the function $f_{2}(\bar{K}, \bar{D})$ in (3.16) is the gradient of the functional (2.1) with respect to $\bar{D}$. By $(3.9), f_{2}(\bar{K}, \bar{D})$ is negative at $\bar{D}>0$ and a small $\bar{K}$, therefore, the optimal adaptation is corner indeed: $\bar{D}=0$. Then the corresponding $\bar{K}$ is interior and is found from $(3.8)$ at $\bar{D}=0$, i.e., from the equation (3.17). Substituting (3.17) into the condition $f_{2}(\bar{K}, \bar{D})<0$, we obtain the inequality (3.18).

The inequality (3.18) always holds when $\widehat{K}$ is small enough. Let us consider the values of $\widehat{K}$ from zero up to some threshold value $\widehat{K}=\widetilde{K}$ that satisfies the equality in (3.18). If it never happens at the given parameters, then the optimal adaptation is always $\bar{D}=0$. The value $\widetilde{K}$ is critical. If $\widehat{K}$ is larger than $\widetilde{K}$, then $f_{2}(\bar{K}, \bar{D})$ becomes positive at $\bar{D}=0$ (because $f_{2}(\bar{K}, 0)$ strictly decreases in $\bar{K}$ at $\bar{D}=0$ ). Therefore, the optimal adaptation $\bar{D}$ should be positive to reach the equality $f_{2}(\bar{K}, \bar{D})=0$. 
Next, the equation (3.17) can be written as

$$
f_{0}(\bar{K})=\frac{(\gamma A)^{1+\mu} \bar{\eta}}{\left(\delta_{P}+\rho\right) \delta_{P}^{\mu}}\left(\frac{\delta+\rho}{\alpha}-\delta\right)
$$

where the function $f_{0}(\bar{K})=\bar{K}^{\alpha-1}\left(A-\bar{K}^{1-\alpha} \frac{\delta+\rho}{\alpha}\right)^{2+\mu}$ strictly decreases in $\bar{K}$. If $\bar{D}>0$ then the equation (3.8) leads to the inequality

$$
f_{0}(\bar{K})=\bar{K}^{\alpha-1}\left(A-\bar{K}^{1-\alpha} \frac{\delta+\rho}{\alpha}\right)^{2+\mu}<\frac{(\gamma A)^{1+\mu} \bar{\eta}}{\left(\delta_{P}+\rho\right) \delta_{P}^{\mu}}\left(\frac{\delta+\rho}{\alpha}-\delta\right) .
$$

Comparing (3.19) and (3.20), we obtain that $\bar{K}>\widehat{K}$. The theorem is proven.

Theorem 1 has important economic implications, which are summarized in the following corollary.

Corollary 3.3. If the optimal adaptation $\bar{D}$ is positive, then the corresponding capital level $\bar{K}$ is larger, the pollution level $\bar{P}$ is higher, and less effort is devoted to abatement (the abatement investment ratio $\bar{B} \bar{K}$ is smaller) compared to the case of no adaptation.

Proof. Indeed, the capital without adaptation is $\bar{K}_{N D}=\widehat{K}$, and $\bar{K}>\widehat{K}$ by the proof of Theorem 1 . The corresponding $\bar{B}_{N D}$ and $\bar{P}_{N D}$ are expressed through $\bar{K}_{N D}$ by formulas (3.10) and (3.12). An elementary analysis of these formulas validates the corollary.

By the theorem on the implicit function, the condition for the uniqueness of positive solutions $\bar{D}$ and $\bar{K}$ is that the determinant of the Jacobean matrix for the nonlinear system (3.14) is non-zero:

$$
\frac{\partial f_{1}(\bar{K}, \bar{D})}{\partial K} \frac{\partial f_{2}(\bar{K}, \bar{D})}{\partial D} \neq \frac{\partial f_{2}(\bar{K}, \bar{D})}{\partial K} \frac{\partial f_{1}(\bar{K}, \bar{D})}{\partial D} .
$$

It turns out that this condition is too complicated for a meaningful interpretation. We can prove the existence of positive solutions $\bar{D}$ and $\bar{K}$ of the nonlinear equations (3.14) at least, when $\bar{D}$ is small, using the linearization of the nonlinear functions

$$
\begin{aligned}
& f_{1}(K, D)=f_{1}(\widehat{K}, 0)+\frac{\partial f_{1}(\widehat{K}, 0)}{\partial K}(K-\widehat{K})+\frac{\partial f_{1}(\widehat{K}, 0)}{\partial D} D \\
& f_{2}(K, D)=f_{2}(\widehat{K}, 0)+\frac{\partial f_{2}(\widehat{K}, 0)}{\partial K}(K-\widehat{K})+\frac{\partial f_{2}(\widehat{K}, 0)}{\partial D} D .
\end{aligned}
$$

However, finding an analytic solution $\bar{D}$ and $\bar{K}$ of these nonlinear equations seems to be impossible because of their complexity. To analyze the sensitivity of the optimal dynamics to given model parameters, including the capital deterioration factor, we rely on numerical simulations. This is the goal of the next section.

\section{Numerical Simulations}

To illustrate the outcomes of analytic modeling, we solve the system of two nonlinear equations (3.8)(3.9) numerically for different ranges of the model parameters. One of our objectives is to estimate the sensitivity of the steady state $(\bar{K}, \bar{C}, \bar{B}, \bar{P}, \bar{D})$ to the capital deterioration factor $\delta$ in order to validate or to revise the policy recommendations given by [4]. A sensitivity analysis is carried out for all economically relevant parameter values. The simulation is done using Excel Solver. The results are provided and discussed in the next two subsections. 


\subsection{Economy with essential pollution}

In this subsection, we investigate the effects of positive capital depreciation compared to the nondepreciation case which is commonly considered in the theoretical literature. Initial data in the first simulation case are based on $[4,19]$. Specifically, we consider the following model parameters: $\rho=0.01$, $\alpha=0.66, \delta_{P}=0.0002, \gamma=0.16, \mu=1, \eta=0.0015, \bar{\eta}=0.003, a=0.001$. The dimensional analysis of all model variables and the plausibility of chosen parameter values are explored in [4]. This data set reflects a common viewpoint of the climate science and presumes that the global environmental selfcleaning capability $\delta_{P}$ is negligible compared to the emission impact factor $\gamma$ and the environmental vulnerability $\eta$. However, we choose the capital depreciation $\delta=0.01$ for both adaptation and productive capitals as opposed to [4] where $\delta$ is zero. We intentionally assume rather small capital depreciation to highlight the great sensitivity of our model to it (a larger more realistic $\delta$ is used in Section 4.2). Recalling that $A$ represents the economic efficiency, we provide simulations for different values of $A$ from 1 to 40 in order to determine the optimal policies for various levels of economic development. In the chosen units of measurement, the US productivity corresponds approximately to $A=25$. The comparative results for the sustainable levels of the optimal capital $K$ and adaptation capital $D$ in cases with and without deterioration are provided in Figures 1-3 for different values $A$. The main insights are the following.

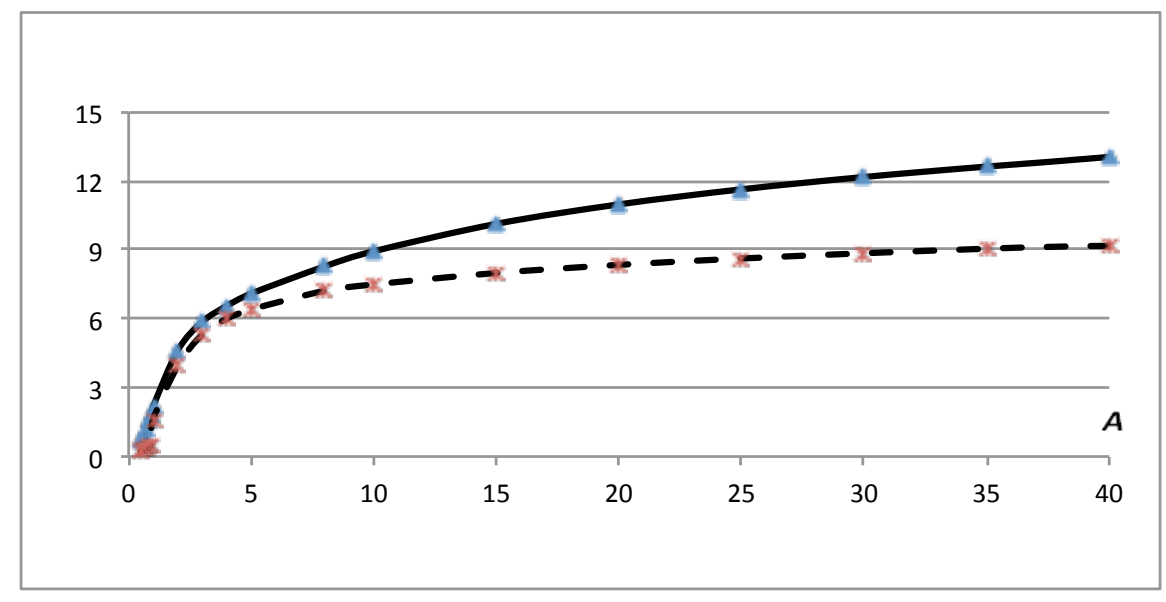

Figure 1. Optimal output $\bar{Y}$ (in logarithmic scale) for various values of the economic efficiency $A$ in two cases: no deterioration, $\delta=0$ (a solid line) and with deterioration (a dashed line) at $\delta=0.01$.

Capital depreciation greatly affects the state of economy. The simulation outcomes demonstrate a significant impact of the capital deterioration on sustainable development. The optimal steady-state levels of the capital $K$, production output $Y$, abatement investment $B$, and other economic characteristics appear to be only $10-40 \%$ of their levels in the case without deterioration. Figure 1 compares the optimal output $Y$ in the logarithmic scale and demonstrates that the difference in $Y$ increases for more productive economies.

No adaptation in inefficient economies. Figures 2 and 3 illustrate the optimal steady-state adaptation capital $D$ with respect to $A$. The optimal adaptation stock $D$ with depreciation remains below the one without depreciation for any productivity $A$. The absence of adaptation for low productivity levels predicted by Theorem 1 clearly appears in both figures. Figure 3 uses the logarithmic scale and the smaller range of $A$ from 0.5 to 5 to demonstrate this effect. One can see the clear (and quite sharp) change in the optimal adaptation level when the economic efficiency $A$ reaches some threshold ( $A$ approaching 1$)$ in the presence of capital depreciation. We cannot simulate this effect at $\delta=0$ because then the nonlinear 


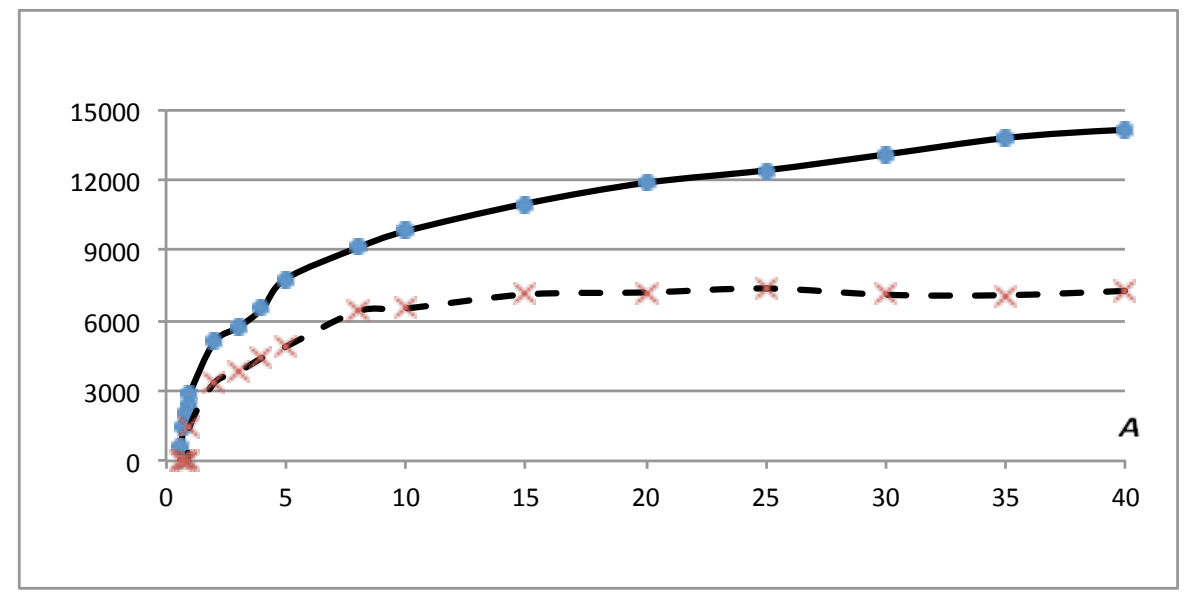

Figure 2. Optimal adaptation capital $\bar{D}$ for various values of $A$ in two cases: no depreciation, $\delta=0$ (a solid line) and with depreciation (a dashed line) at $\delta=0.01$.

system (3.8)-(3.9) becomes ill-posed at small values of $A$ and its numeric solution (shown with the solid curve in Fig.3) is economically impossible (the calculated optimal ratio $D / K$ is $100-400$ for $A<1$ ). It happens because, in the absence of capital deterioration, the steady-state adaptation $D$ appears to be a free force to compensate the economic inefficiency. Namely, the adaptation investment $\bar{I}_{D}=\delta \bar{D}$ is zero at $\delta=0$ and does not affect the distribution equation (3.3). In other words, we do not need to invest to keep any desired steady-state adaptation level $\bar{D}$. Then, the unknown $\bar{D}$ appears in the equations (3.8.)-(3.9) in the term $e^{-a \bar{D}}$ only, which leads to instability of their numeric solution. In summary, the presence of the adaptation investment $\delta \bar{D}$ in the distribution equation (3.3) at $\delta>0$ is not only reasonable economically but it also regularizes numeric solution of the nonlinear system (3.3)-(3.7).

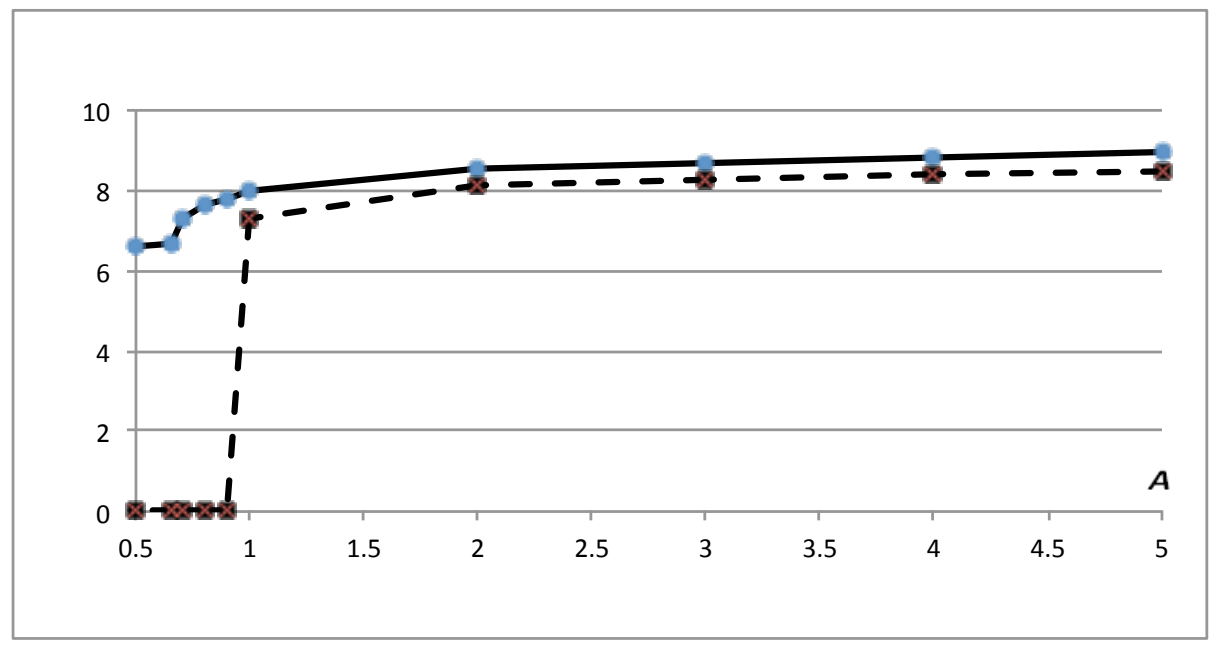

FiguRE 3. The optimal adaptation capital (in logarithmic scale) for $0.5<A<5$ without deterioration (solid line) and with deterioration at $\delta=0.01$ (dashed line).

Welfare level. It is interesting to compare the welfare level in two economies with and without adaptation in the case of positive capital depreciation. To calculate the sustainable welfare level, let us assume 
that initial conditions $K_{0}, D_{0}, P_{0}$ in (2.4)-(2.6) are $K_{0}=\bar{K}, D_{0}=\bar{D}$ and $P_{0}=\bar{P}$. Then, substituting $\bar{C}, \bar{D}$ and $\bar{P}$ into (2.1), the sustainable welfare level is given by:

$$
W=\frac{1}{\rho}\left(\ln \bar{C}-\eta(\bar{D}) \frac{\bar{P}^{\mu+1}}{(\mu+1)}\right)
$$

By (4.1), the pollution level $\bar{P}$ is larger with adaptation, but this negative effect is offset in (4.1) by a smaller environmental vulnerability value $\eta(\bar{D})$. It turns out in our numerical experiments that the calculated welfare level increases with adaptation than without (up to $50 \%$ of the original value when $A \geqslant 20$, i.e., the economy is strong). Because of extremely polluted environment, the sustainable welfare level is negative. A less extreme simulation case is considered in the next section.

\subsection{Less vulnerable economy}

The second simulation case describes a more favorable environmental situation. Namely, we take the same values $\rho=0.01, \alpha=0.66, \delta_{P}=0.0002, a=0.001$, but with a much smaller polluting economy $\gamma=0.0001$, less damaging pollution $\mu=0.1$, and wider range of environmental vulnerability $\eta=0.005$, $\bar{\eta}=0.01$. For those parameters, we should also choose a more realistic capital depreciation rate rather than $\delta=0.01$ as above. The reason is that the whole steady state of an economy is highly sensitive to the capital depreciation rate. If we keep the same $\delta=0.01$, then the calculated steady-state adaptation more than 1000 times larger and steady-state capital would be 20-50 times larger compared to the case $\delta=0.05$. So, we select the depreciation rate $\delta=0.05$ that delivers more realistic simulation results. The chosen dataset reflects an optimistic case of an economy that can survive just with modest emission abatement because the environmental selfcleaning capability $\delta_{P}$ is larger than the pollution emission impact factor $\gamma$. As a result, simulation outcomes are less dramatic than in the previous case.

Capital depreciation. Our numerical experiments show that the optimal capital stock $\mathrm{K}$ at $\delta=0.05$ is smaller than $1 \%$ of its level for $\delta=0$. Meanwhile, the consumption $\mathrm{C}$ is $\simeq 1.3 \%$ and the output $\mathrm{Y}$ is $\simeq 3 \%$ of their levels without depreciation. For comparison, we repeated the simulation in this case for $\delta=0.01$, then the optimal capital $K$ level is $13-30 \%$, the output $Y$ is $25-60 \%$, and the consumption $C$ is $17-40 \%$ of their levels without deterioration, which is comparable to the case of Section 4.1 . So, the impact of capital deterioration on sustainable development remains strong in the case of a less vulnerable economy.

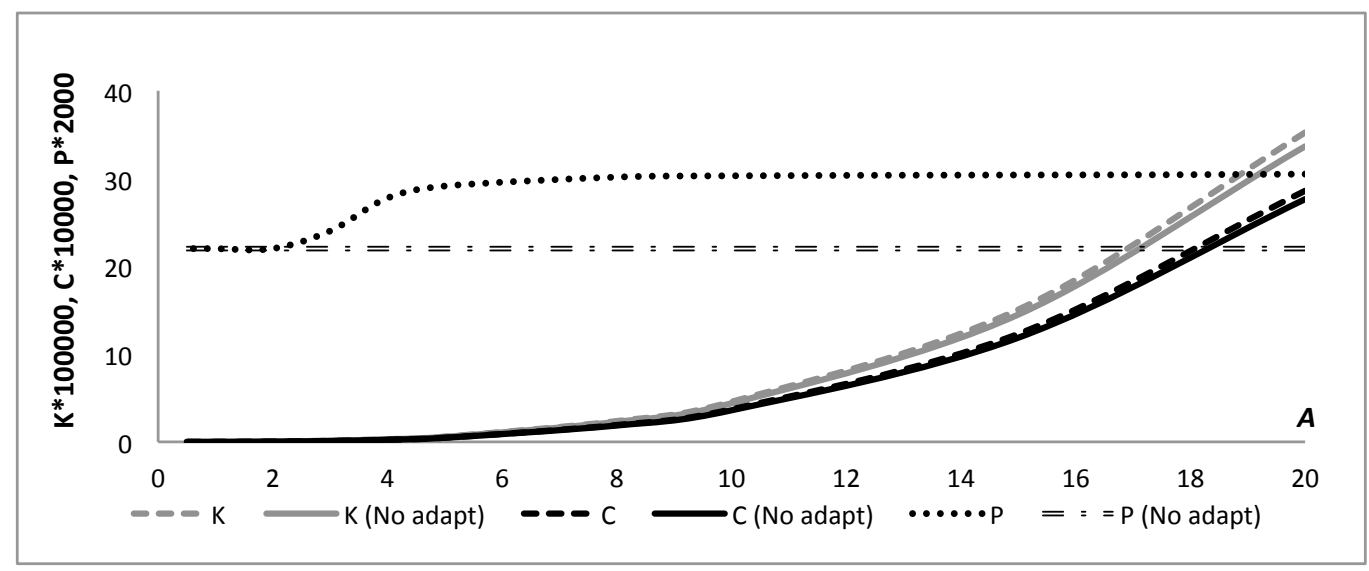

Figure 4. Consumption $\bar{C}$, capital $\bar{K}$, and pollution level $\bar{P}$ at $\delta=0.05$ in two cases: (a) with adaptation and abatement, (b) abatement only. 
Adaptation level versus Abatement? Figures 4 and 5 illustrate the impact of optimal adaptation and abatement investments on the economy with the capital deterioration factor $\delta=5 \%$. The simulation outcomes demonstrate that the adaptation capital $D$ is in the range $0-2 \%$ of the physical capital $K$ at different values of the economic efficiency $A$. The adaptation capital $D$ is zero at $A<2$ and monotonically increases for larger values of $A$. Figure 4 shows that an increase in the pollution level $P$ due to the presence of adaptation is more essential (up to 40\%) as compared to the increase of the economy size (both capital $K$ and consumption $C$ differ by less than $4 \%$ from their levels without adaptation).

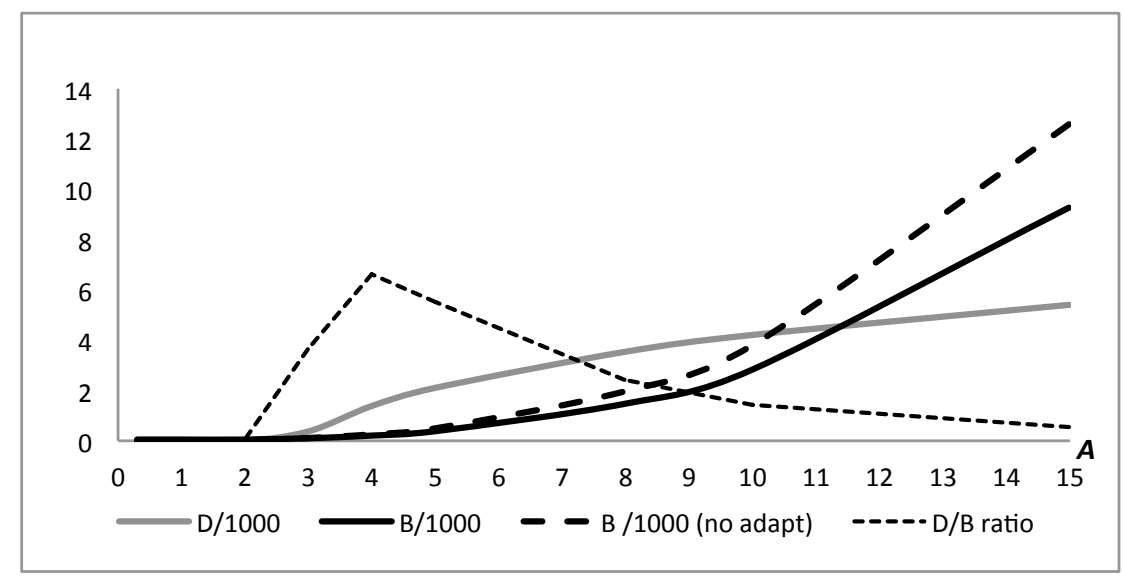

FiguRE 5. Optimal adaptation $\bar{D}$, abatement $\bar{B}$, and adaptation-abatement ratio $\bar{D} / \bar{B}$ for various values of the economic efficiency $A$

Figure 5 shows that both adaptation $D$ and abatement $B$ increase in absolute units, but it also reveals that $B$ increases faster. The abatement $B$ is $30-35 \%$ larger without adaptation than in the case with adaptation. The most interesting effect is that the optimal ratio $D / B$ between adaptation and abatement has an inverted U-shape (a dotted curve in Fig. 5). The ratio $D / B$ increases when the economic efficiency $A$ increases from 2 to $\simeq 4$, and then decreases when $A$ increases further. Specifically, the optimal ratio $D / B$ changes from its maximal value of 6.6 at $A=4$ to only 0.3 at $A=20$.

Welfare level. The sustainable welfare level (4.1) in this simulation case is positive and is only $0.6 \%$ higher with adaptation than without adaptation. The increase in the pollution level is strong (about $40 \%$ ) and is compensated in the welfare formula (4.1) by a smaller vulnerability.

\section{Conclusion}

This paper significantly extends and clarifies the result of [4] that the optimal policy mix of adaptation and abatement depends on the country's economic potential in the case of positive capital depreciation. Specifically, investing into adaptation measures becomes profitable only starting with a certain threshold value of the economic efficiency. So, in the case of a poor country, the optimal environmental policy may be no adaptation to climate change at all. In the case of a developed country, the optimal adaptation/abatement ratio remains rather low. The maximum adaptation efforts (in terms of the adaptation/abatement ratio) should be done by countries at a certain intermediate stage of development.

The obtained analytic results and numeric simulation also demonstrate that the impact of capital depreciation on the optimal long-term sustainable growth is quite essential and cannot be ignored in designing optimal policies to combat climate change. We conclude that our modeling framework is applicable for making practical policy recommendations only with a proper estimation of the deterioration of physical and adaptation capital. 
Acknowledgements. The authors express their gratitude to the participants of 4th Workshop on Game Theory in Energy, Resources and Environment (November 2012, University of Montréal, Québec). The financial support of NSF DMS 1009197 and the Lhoist Berghmans Chair at CORE allowed the co-authors to meet and finish this paper.

\section{References}

[1] S. Agrawala, F. Bosello, C. Carraro, E. de Cian, E. Lanzi. Adapting to climate change: costs, benefits, and modelling approaches. International Review of Environmental and Resource Economics, 5 (2011), 245-284.

[2] R.J. Barro, X. Sala-i-Martin . Economic Growth, NewYork, McGraw Hill, (1995).

[3] F. Bosello, C. Carraro, E. de Cian. Climate policy and the optimal balance between mitigation, adaptation and unavoided damage. Climate Change Economics, 1 (2010), no. 2, 71-92.

[4] Th. Bréchet, N. Hritonenko, Yu. Yatsenko. Adaptation and mitigation in long-term climate policy. Environmental and Resource Economics, 55 (2013), 217-243.

[5] L. Bretschger, S. Valente. Climate change and uneven development. The Scandinavian Journal of Economics, 113 (2011), 825-845

[6] S. Buob, G. Stephan. To mitigate or to adapt: how to combat with global climate change. European Journal of Political Economy, 27 (2011), 1-16.

[7] M.R. Caputo. Foundations of Dynamic Economic Analysis: Optimal Control Theory and Applications, Cambridge University Press, New York, (2005).

[8] C. Corduneanu. Integral Equations and Applications. Cambridge University Press, Cambridge UK, (1991).

[9] K. De Bruin, R. Dellink, aR. Tol. AD-DICE: an implementation of adaptation in the DICE model. Climatic Change, 95 (2009), no. 1, 63-81.

[10] G. Economides, A. Philippopoulos. Growth enhancing policy is the means to sustain the environment. Review of Economic Dynamics, 11 (2008), 207-219.

[11] R. Goetz, N. Hritonenko, A. Xabadia, ÊYu. Yatsenko. Forest management for timber and carbon sequestration in the presence of climate change: The case of Pinus Sylvestris. Ecological Economics, 88 (2013), 86-96.

[12] N. Hritonenko, Yu. Yatsenko. Turnpike properties of optimal delay in integral dynamic models. Journal of Optimization Theory and Applications, 127 (2005), 109-127.

[13] N. Hritonenko, Yu. Yatsenko. Technological innovations, economic renovation, and anticipation effects. Journal of Mathematical Economics, 46 (2010), 1064-1078.

[14] N. Hritonenko, Yu. Yatsenko. Energy substitutability and modernization of energy-consuming technologies. Energy Economics, 34 (2012), 1548-1556.

[15] N. Hritonenko, Yu. Yatsenko. Modeling of environmental adaptation: amenity vs productivity and modernization. Climate Change Economics, 4 (2013) no.2, 1-24.

[16] IPCC (2007). Climate Change 2007, Fourth Assessment Report of the Intergovernmental Panel on Climate Change, Cambridge University Press.

[17] L.E. Jones, R.E. Manuelli. Endogenous policy choice: the case of pollution and growth. Review of Economic Dynamics, 4 (2001), 369-405.

[18] S. Kane, J.F. Shogren. Linking adaptation and mitigation in climate change policy. Climatic Change, 45 (2000), 75-102.

[19] D.G. Luenberger. Introduction to Dynamic Systems: Theory, Models, and Applications. John Wiley and Sons, New York, (1979).

[20] W.D. Nordhaus. A Question of Balance: Weighing the Options on Global Warming Policies. Yale University Press, New Haven \& London, (2008).

[21] C. Rosenzweig, M.L. Parry. Potential impact of climate change on world food supply. Nature, 367 (1994), $133-138$.

[22] S. Shalizi, F. Lecocq. To mitigate or to adapt; is that the question? Observations on an appropriate response to the climate change challenge to development strategies. The World Bank Research Observer, 2 (2009), 1-27.

[23] S. Smulders, R. Gradus. Pollution abatement and long-term growth. European Journal of Political Economy, 12 (1996), 505-532.

[24] N. Vellinga. Multiplicative utility and the influence of environmental care on the short-term economic growth rate. Economic Modelling, 16 (1999), 307-330. 